INT J COMPUT COMMUN, ISSN 1841-9836

Vol.7 (2012), No. 4 (November), pp. 594-605

\title{
Control Schemes for a Quadruple Tank Process
}

\author{
A. Abdullah, M. Zribi
}

\author{
Ali Abdullah, Mohamed Zribi \\ Electrical Engineering Department \\ Kuwait University \\ P. O. Box 5969, Safat-13060, Kuwait \\ E-mail: ali.abdullah@ku.edu.kw \\ E-mail: mohamed.zribi@ku.edu.kw
}

\begin{abstract}
This paper deals with the control of a quadruple tank process. A gain scheduling controller, a linear parameter varying controller and an input-output feedback linearization controller are proposed for the quadruple tank process. The derivation of the three control schemes is presented in details. Moreover, the proposed control schemes are implemented using an experimental setup. The experimental results indicate that the developed control schemes work well and are able to regulate the output of the process to its desired value. Additionally, the implementation results demonstrate that the input-output feedback linearization controller gave the best performance.
\end{abstract}

Keywords: quadruple tank process, gain scheduling control, linear parameter varying control, input-output feedback linearization control.

\section{Introduction}

The quadruple tank process is a highly nonlinear system which has been used to test different multivariable control schemes. Several controllers were designed for this process. For example, a decentralized proportional integral (PI) controller [1,2], a decentralized PI controller with sliding mode features [3], a decoupled proportional integral and derivative (PID) controller [4], an internal model controller [5], a model predictive controller [6,7], a quantitative feedback controller [8] and an $H_{\infty}$ controller [9] were proposed for the control of the quadruple tank process. These control schemes were designed using the linearized model of the quadruple tank process around different operating points. Therefore, these controllers can not guarantee good performances of the controlled system over the whole operating range of the quadruple tank process because of the inherent nonlinearities of the quadruple tank process.

In order to achieve good performances over the whole operating range of the quadruple tank process, other control techniques were reported in the literature. Nonlinear model predictive controllers where designed in $[10,11]$ for the process. In [12], a sliding mode controller was designed and implemented on the process. However, it should be noted that a singularity is encountered when using this controller. The singularity occurs when one of the four tanks is empty. Hence, the proposed controller can not be implemented in such case. In [13], a linear decentralized PI controller was designed based on the approximated nonlinear model of the process over a selected range of operation of the process. The simulation results show a good tracking behavior over the selected operating range. On the other hand, the work in [14] dealt with the design of a gain scheduling PI controller for the process; the gain scheduling design is done according to the operating input voltages.

In this paper, three well-known controllers consisting of a gain scheduling control [15-17], a linear parameter varying control [18-21] and an input-output feedback linearization control [22,23] are designed and implemented to control the water levels in the quadruple tank process over the whole range of operation of the process. Moreover, an integral action is included in the three control schemes in order to achieve good tracking performances [22]. The implementation results 
are presented to show the effectiveness of the proposed control schemes.

The paper is organized as follows. The dynamic model of the process and the control objective of the paper are presented in sections 2 and 3 respectively. A gain scheduling controller, a linear parameter varying controller, and an input-output feedback linearization controller are designed in sections 4, 5 and 6 respectively. The experimental results are presented and discussed in section 7 . Finally, some concluding remarks are given in section 8 .

\section{The dynamic model of the quadruple tank process}

A schematic diagram and a picture of the quadruple tank process are shown in Fig. 1. The system consists of four cylindrical tanks and two pumps; these pumps are connected to valves for water distribution. Pump 1 is used to distribute water from the water reservoir to tanks 1 and 4, while pump 2 is used to distribute water to tanks 2 and 3. Four pressure sensors which are located at the bottom of each tank are used to measure the water levels in the tanks.
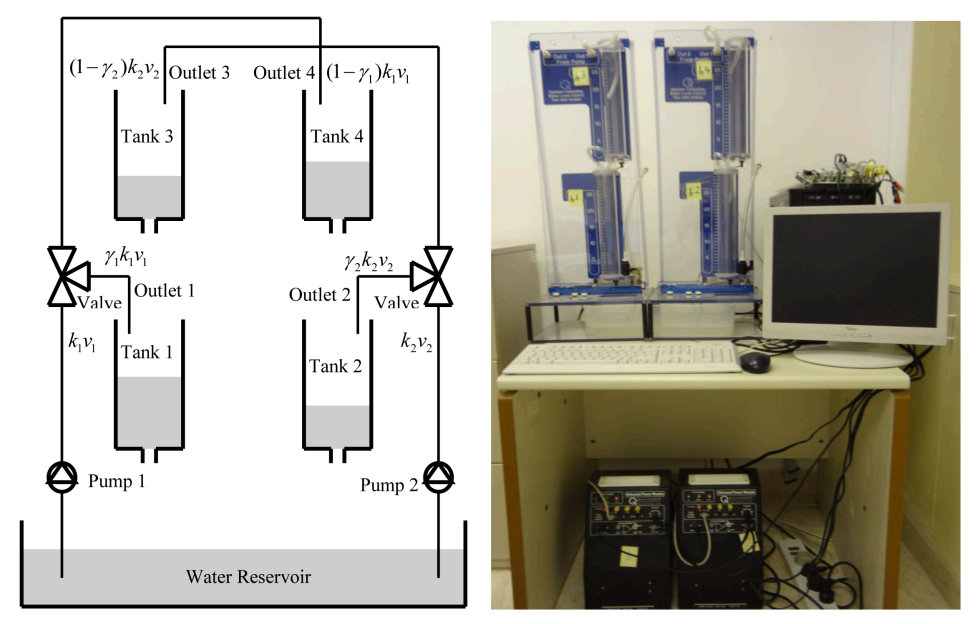

Figure 1: A schematic diagram (left) and a picture (right) of the quadruple tank process

The dynamic model of the quadruple tank process can be written as [1]:

$$
\left[\begin{array}{c}
\dot{h_{1}} \\
\dot{h_{2}} \\
\dot{h_{3}} \\
\dot{h_{4}}
\end{array}\right]=\underbrace{\left[\begin{array}{c}
-p_{1} \sqrt{h_{1}}+p_{2} \sqrt{h_{3}} \\
-p_{4} \sqrt{h_{2}}+p_{5} \sqrt{h_{4}} \\
-p_{7} \sqrt{h_{3}} \\
-p_{9} \sqrt{h_{4}}
\end{array}\right]}_{f(h)}+\underbrace{\left[\begin{array}{cc}
p_{3} & 0 \\
0 & p_{6} \\
0 & p_{8} \\
p_{10} & 0
\end{array}\right]}_{B}[\underbrace{\left[\begin{array}{l}
v_{1} \\
v_{2}
\end{array}\right]}_{v}, y=\underbrace{\left[\begin{array}{llll}
1 & 0 & 0 & 0 \\
0 & 1 & 0 & 0
\end{array}\right]}_{C} \underbrace{\left[\begin{array}{c}
h_{1} \\
h_{2} \\
h_{3} \\
h_{4}
\end{array}\right]}_{h}
$$

where $p_{1}=a_{1} \sqrt{2 g} / A_{1}, p_{2}=a_{3} \sqrt{2 g} / A_{1}, p_{3}=\gamma_{1} k_{1} / A_{1}, p_{4}=a_{2} \sqrt{2 g} / A_{2}, p_{5}=a_{4} \sqrt{2 g} / A_{2}$, $p_{6}=\gamma_{2} k_{2} / A_{2}, p_{7}=a_{3} \sqrt{2 g} / A_{3}, p_{8}=\left(1-\gamma_{2}\right) k_{2} / A_{3}, p_{9}=a_{4} \sqrt{2 g} / A_{4}$, and $p_{10}=\left(1-\gamma_{1}\right) k_{1} / A_{4}$. The variables and the parameters of the process are the water level $h_{i}$, the cross-section area $A_{i}$, the outlet cross-section area $a_{i}$ of tank $i(i=1,2, \ldots, 4)$, the voltage $v_{j}$ applied to pump $j$, the constant gain $k_{j}$ of pump $j$, the constant of the valve $\gamma_{j}$ connected to pump $j(j=1,2)$. The output of the system is $y$ and the gravitational acceleration is $g$. 


\section{The control objective of the paper}

The objective of the paper is to design control schemes such that the outputs of the process, i.e. $h_{1}$ and $h_{2}$, asymptotically converge to the desired levels $h_{1}^{o}$ and $h_{2}^{o}$. The steady state value of the applied voltage vector $v^{o}=\left[v_{1}^{o}, v_{2}^{o}\right]^{T}$ which can maintain the water level vector at $h^{o}=\left[h_{1}^{o}, h_{2}^{o}, h_{3}^{o}, h_{4}^{o}\right]^{T}$ must satisfy the following equilibrium equations:

$-p_{1} \sqrt{h_{1}^{o}}+p_{2} \sqrt{h_{3}^{o}}+p_{3} v_{1}^{o}=0,-p_{4} \sqrt{h_{2}^{o}}+p_{5} \sqrt{h_{4}^{o}}+p_{6} v_{2}^{o}=0,-p_{7} \sqrt{h_{3}^{o}}+p_{8} v_{2}^{o}=0,-p_{9} \sqrt{h_{4}^{o}}+p_{10} v_{1}^{o}=0$

Clearly, equations (2) imply that one can only select the values of two water levels. For instance, if we select the values of $h_{1}^{o}$ and $h_{2}^{o}$ (since they represent the desired outputs of the system) then it can be shown that the steady state values of $h_{3}^{o}$ and $h_{4}^{o}$ must satisfy the following matrix equation:

$$
\left[\begin{array}{c}
\sqrt{h_{3}^{o}} \\
\sqrt{h_{4}^{o}}
\end{array}\right]=\left[\begin{array}{cc}
p_{2} / p_{1} & \left(p_{3} p_{9}\right) /\left(p_{1} p_{10}\right) \\
\left(p_{6} p_{7}\right) /\left(p_{4} p_{8}\right) & p_{5} / p_{4}
\end{array}\right]^{-1}\left[\begin{array}{c}
\sqrt{h_{1}^{o}} \\
\sqrt{h_{2}^{o}}
\end{array}\right]
$$

It should be noted that the inverse of the matrix in (3) exists when $p_{2} p_{5} p_{8} p_{10} \neq p_{3} p_{6} p_{7} p_{9}$, which is equivalent to $\gamma_{1}+\gamma_{2} \neq 1$.

\section{Design of a gain scheduling controller}

In this section, a gain scheduling controller is designed using the classical approach of gain scheduling design [15]. At first, the nonlinear dynamic model of the process under a linear controller is linearized around several operating points. Then, a linear controller is designed at each operating point to meet the required specifications. Finally, the resulting linear controllers are interpolated according to the water levels $h_{1}$ and $h_{2}$ to produce a single gain scheduling controller. The obtained controller is used to regulate the output of the process from one operating point to another operating point.

Consider the following state feedback integral controller:

$$
v=-K_{h} h-K_{\sigma} \sigma-K_{e} e, \quad \dot{\sigma}=e=r-y
$$

where $r=\left[r_{1}, r_{2}\right]^{T}$ is the reference vector and $K_{h}, K_{\sigma}$, and $K_{e}$ are the gains of the controller. The closed loop system when using the controller (4) into the model of the process given by (1) is such that:

$$
\dot{h}=f(h)-B\left[\left(K_{h}-K_{e} C\right) h+K_{\sigma} \sigma+K_{e} r\right], \quad \dot{\sigma}=r-C h, \quad y=C h
$$

When $r=\left[h_{1}^{o}, h_{2}^{o}\right]^{T}$, the closed loop system (5) has an equilibrium point at $\left(h^{o}, \sigma^{o}\right)$ where $h^{o}$ satisfies (3), $e=0$, and $\sigma^{o}=-K_{\sigma}^{-1}\left[K_{h} h^{o}+v^{o}\right]$ provided that the matrix $K_{\sigma} \in R^{2 \times 2}$ is nonsingular. To obtain a linear system, we linearize the closed loop system (5) about $\left(h^{o}, \sigma^{o}\right)$ to yield:

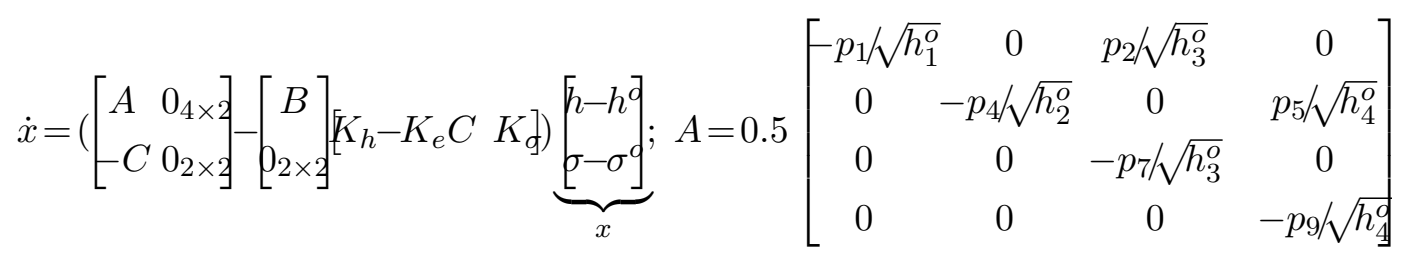

where $A=\partial f(h) /\left.\partial h\right|_{h=h^{o}}$. The gains $K_{h}, K_{\sigma}$, and $K_{e}$ are designed using the linearized feedback system (6) such that all closed loop poles lie inside a prescribed region shown in Fig. 2. Note 
that by placing the closed loop poles inside the shaded region, we ensure that good responses are obtained. This is the case because by placing the closed loop poles inside this region results in i) a minimum decay rate $\tau$, ii) a minimum damping ratio $\zeta=\cos (\varphi)$, and iii) acceptable control gains penalized by $\rho$.

Now, assume that the output of the process needs to be regulated to the $i$ th operating point $\left(h_{1}^{o^{i}}, h_{2}^{o^{i}}\right)$ and then to the $(i+1)$ th operating point $\left(h_{1}^{o^{i+1}}, h_{2}^{o^{i+1}}\right)$. To achieve this task, the linearized feedback system given by (6) is used to obtain a set of gains $\left\{K_{h}^{l}, K_{\sigma}^{l}, K_{e}^{l}\right\},(l=1, \ldots, 4)$, which are designed to meet the above mentioned specifications at each of the following operating points: $\left(h_{1}^{o^{i}}, h_{2}^{o^{i}}\right),\left(h_{1}^{o^{i}}, h_{2}^{o^{i+1}}\right),\left(h_{1}^{o^{i+1}}, h_{2}^{o^{i}}\right)$ and $\left(h_{1}^{o i+1}, h_{2}^{o^{i+1}}\right)$, respectively. The corresponding set of controllers are given by $v^{l}=-K_{h}^{l} h-K_{\sigma}^{l} \sigma-K_{e}^{l} e$ for $l=1,2, \ldots, 4$. Using the bilinear interpolating method [16], these control outputs are interpolated according to the water levels $h_{1}$ and $h_{2}$ to produce the following gain scheduling controller:

$$
v=\eta_{1} v^{1}+\eta_{2} v^{2}+\eta_{3} v^{3}+\eta_{4} v^{4}
$$

where $\eta_{1}=\left(h_{1}^{i+1}-h_{1}\right)\left(h_{2}^{o i+1} h_{2}\right) /\left(\left(h_{1}^{o^{i+1}} h_{1}^{o^{i}}\right)\left(h_{2}^{o^{i+1}} h_{2}^{o^{i}}\right)\right), \eta_{2}=-\left(h_{1}^{o^{i+1}} h_{1}\right)\left(h_{2}^{o^{i}} h_{2}\right) /\left(\left(h_{1}^{o^{i+1}} h_{1}^{o^{i}}\right)\left(h_{2}^{o^{i+1}} h_{2}^{o^{i}}\right)\right)$, $\eta_{3}=-\left(h_{1}^{o^{i}} h_{1}\right)\left(h_{2}^{o i+1} h_{2}\right) /\left(\left(h_{1}^{o^{i+1}}-h_{1}^{o^{i}}\right)\left(h_{2}^{o+1}-h_{2}^{o^{i}}\right)\right)$ and $\eta_{4}=\left(h_{1}^{o^{i}} h_{1}\right)\left(h_{2}^{o^{i}} h_{2}\right) /\left(\left(h_{1}^{o^{i+1}}-h_{1}^{o^{i}}\right)\left(h_{2}^{o^{i+1}}-h_{2}^{o^{i}}\right)\right)$.

The controller given by (7) is applied to the quadruple tank process to regulate $\left(h_{1}, h_{2}\right)$ to the operating point $\left(h_{1}^{o^{i}}, h_{2}^{o^{i}}\right)$ and then to the next operating point $\left(h_{1}^{o^{i+1}}, h_{2}^{o^{i+1}}\right)$. It should be noted that the controller (7) does not guarantee the stability and the performance of the system over the whole range of operation of process; this drawback can be overcome by using the LPV approach developed in the next section.

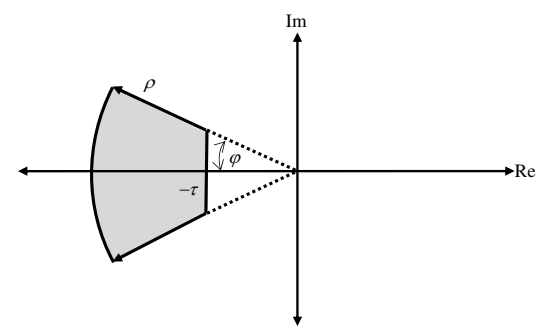

Figure 2: Pole-placement region

\section{Design of a linear parameter varying controller}

This section presents the design of a linear parameter varying (LPV) controller for the quadruple tank process. In order to design an LPV controller, the process model (1) needs to be written as a quasi-LPV model. To achieve this goal, a standard polynomial fitting technique [26] is used to approximate the nonlinear terms $\sqrt{h_{i}}$ with ${ }_{i} h_{i}$ for $0 \leq h_{i} \leq \bar{h}_{i}=30 \mathrm{~cm}$, where ${ }_{i}$ is obtained as ${ }_{i}=0.583-4.036 \times 10^{-2} h_{i}+1.73 \times 10^{-3} h_{i}^{2}-3.659 \times 10^{-5} h_{i}^{3}+2.981 \times 10^{-7} h_{i}^{4}$ for $i=1, \ldots, 4$. It can be shown that the parameters $i$ are bounded such that $0.1={ }_{i} \leq_{i} \leq{ }_{i}=0.6$. Notice that the parameter vector $=[1,2,3,4]^{T}$ is varying inside a hyper-rectangle region with $2^{4}$ vertices defined as $\Lambda_{j} \in\left\{\left(v_{1, j}, \ldots, v_{4, j}\right) \mid v_{i, j} \in\left\{{ }_{i},{ }_{i}\right\}\right\}$ for $j=1, \ldots, 2^{4}$, where $v_{i, j} \in R$ is the $i$ th element of $\Lambda_{j} \in R^{4}$. 
Therefore, the process model (1) can be written in the following quasi-LPV form:

$$
\left[\begin{array}{l}
\dot{h_{1}} \\
\dot{h_{2}} \\
\dot{h_{3}} \\
\dot{h_{4}}
\end{array}\right]=\underbrace{\left[\begin{array}{cccc}
-p_{11} & 0 & p_{23} & 0 \\
0 & -p_{42} & 0 & p_{54} \\
0 & 0 & -p_{73} & 0 \\
0 & 0 & 0 & -p_{94}
\end{array}\right]}_{A()} \underbrace{\left[\begin{array}{c}
h_{1} \\
h_{2} \\
h_{3} \\
h_{4}
\end{array}\right]}_{h}+\underbrace{\left[\begin{array}{cc}
p_{3} & 0 \\
0 & p_{6} \\
0 & p_{8} \\
p_{10} & 0
\end{array}\right]}_{B} \underbrace{\left[\begin{array}{l}
v_{1} \\
v_{2}
\end{array}\right]}_{v}, y=\underbrace{\left[\begin{array}{llll}
1 & 0 & 0 & 0 \\
0 & 1 & 0 & 0
\end{array}\right]}_{C} \underbrace{\left[\begin{array}{c}
h_{1} \\
h_{2} \\
h_{3} \\
h_{4}
\end{array}\right]}_{h}
$$

Let the LPV state feedback integral controller be such that:

$$
v=-\underbrace{\left(\bar{K}_{h_{0}}+\sum_{i=1}^{4}{ }_{i} \bar{K}_{h i}\right)}_{\bar{K}_{h}()} h-\bar{K}_{\sigma} \sigma-\bar{K}_{e} e, \quad \dot{\sigma}=e=r-y
$$

where the controller gains $\bar{K}_{h_{l}}(l=0,1, \ldots, 4), \bar{K}_{\sigma}$, and $\bar{K}_{e}$ are designed such that the required specifications are met for all admissible values of the parameter vector .

The closed loop dynamic model of the process when using (8) and (9) is such that:

$\dot{\bar{x}}=(\underbrace{\left[\begin{array}{cc}A() & 0_{4 \times 2} \\ -C & 0_{2 \times 2}\end{array}\right]}_{\bar{A}()}-\underbrace{\left[\begin{array}{c}B \\ 0_{2 \times 2}\end{array}\right]}_{\bar{B}} \underbrace{\left[\begin{array}{cc}\bar{K}_{h e}() & \bar{K}_{\sigma}\end{array}\right]}_{\bar{K}()}) \underbrace{\left[\begin{array}{c}h \\ \sigma\end{array}\right]}_{\bar{x}}+\underbrace{\left[\begin{array}{c}-B \bar{K}_{e} \\ I_{2 \times 2}\end{array}\right]}_{B_{c}} r, \quad y=\underbrace{\left[\begin{array}{cc}C & 0_{2 \times 2}\end{array}\right]}_{C_{c}} \bar{x}(10)$

The gain $\bar{K}()=\left[\begin{array}{ll}\bar{K}_{h e}() & \bar{K}_{\sigma}\end{array}\right]$ is designed to guarantee the stability of the closed loop system for any possible trajectory. To obtain a good performance of the closed loop system, the closed loop poles of the system (10) (at the $2^{4}$ vertices) are forced to lie in the left half of the complex plane and inside the region shown in Fig. 2. This objective is achieved by considering the LPV gain $L()$ such that $L()=L_{0}+\sum_{i=1}^{4}{ }_{i} L_{i}=\bar{K}() P$, where $P$ is a positive definite matrix. Then, the following set of LMIs [20] are solved for $P$ and $L_{l}(l=0,1, \ldots, 4)$ :

$$
\begin{gathered}
M\left(\Lambda_{j}\right)+M^{T}\left(\Lambda_{j}\right)+2 \tau P<0 \\
{\left[\begin{array}{cc}
-\rho P & M\left(\Lambda_{j}\right) \\
M^{T}\left(\Lambda_{j}\right) & -\rho P
\end{array}\right]<0\left[\begin{array}{ll}
\sin (\varphi)\left(M\left(\Lambda_{j}\right)+M^{T}\left(\Lambda_{j}\right)\right) & \cos (\varphi)\left(M\left(\Lambda_{j}\right)-M^{T}\left(\Lambda_{j}\right)\right) \\
\cos (\varphi)\left(M^{T}\left(\Lambda_{j}\right)-M\left(\Lambda_{j}\right)\right) & \sin (\varphi)\left(M\left(\Lambda_{j}\right)+M^{T}\left(\Lambda_{j}\right)\right)
\end{array}\right]<0(11)}
\end{gathered}
$$

where $M\left(\Lambda_{j}\right)=\bar{A}\left(\Lambda_{j}\right) P-\bar{B} L\left(\Lambda_{j}\right)$ for $j=1,2,3, \ldots, 2^{4}$. Once the matrices $P$ and $L_{l}(l=$ $0,1, \ldots, 4)$ are obtained using any available software such as the LMI Control Toolbox [25], the controller gains $\bar{K}_{h_{0}}, \bar{K}_{h_{l}}$ and $\bar{K}_{\sigma}$ are calculated using the matrix equations $\left[\begin{array}{cc}\bar{K}_{h_{0}}-\bar{K}_{e} C & \bar{K}_{\sigma}\end{array}\right]=$ $L_{0} P^{-1}$ and $\left[\begin{array}{cc}\bar{K}_{h l} & 0_{2 \times 2}\end{array}\right]=L_{l} P^{-1}$ for $l=1, \ldots, 4$, where the gain $\bar{K}_{e}$ is designed to improve the closed-loop performance of the system.

The following proposition gives the main result of this section.

Proposition 1. The LPV controller (9) with gains obtained using (11) guarantees the stability of the closed loop system (10) and the regulation of the system output y to its desired value over the whole range of operation of the process. Furthermore, the closed loop poles at each vertex of the scheduling variable are located inside the region shown in Fig. 2.

Proof: Consider the matrix equation $\bar{K}()=L() P^{-1}$. Let $V=\bar{x}^{T} P^{-1} \bar{x}$ be a Lyapunov function candidate for the system (10), then the time derivative of $V$ along the trajectories of the system while assuming that $r=0$ is given by $\dot{V}=\left(P^{-1} \bar{x}\right)^{T}\left[\bar{A}() P-\bar{B} L()+P \bar{A}^{T}()-L()^{T} \bar{B}^{T}\right]\left(P^{-1} \bar{x}\right)=$ 
$\left(P^{-1} \bar{x}\right)^{T}\left[M()+M^{T}()\right]\left(P^{-1} \bar{x}\right)$. Given that $M\left(\Lambda_{j}\right)+M^{T}\left(\Lambda_{j}\right)<-2 \tau P<0$ at each vertex $\Lambda_{j}$. then $M()+M^{T}()<-2 \tau P<0$ for all admissible values of which implies that $\dot{V}<0$. Therefore, the LPV controller (9) guarantees the stability of the closed loop system (10). Furthermore, the integral term in the LPV controller (9) ensures the regulation of the output to its desired level. Moreover, the LMIs (11) ensure that the closed loop poles at each vertex $\Lambda_{j}$ are located inside the region shown in Fig. 2 (see [20]).

The LPV control design approach is computationally intensive because the LMIs (11) need to be solved at the $2^{4}$ vertices. Therefore, the following section presents a controller which is less computationally intensive than the proposed LPV controller.

\section{Design of an input-output feedback linearization controller}

This section deals with the design of an input-output feedback linearization controller for the quadruple tank process.

Let the input-output feedback linearization controller be such that:

$v_{1}=\left(1 / p_{3}\right)\left[\kappa_{1} e_{1}+\kappa_{2} \int_{0}^{t} e_{1} d \dot{t}+p_{1} \sqrt{h_{1}}-p_{2} \sqrt{h_{3}}\right], v_{2}=\left(1 / p_{6}\right)\left[\kappa_{3} e_{2}+\kappa_{4} \int_{0}^{t} e_{2} d \dot{t}+p_{4} \sqrt{h_{2}}-p_{5} \sqrt{h_{4}}\right]$

where $e_{1}=r_{1}-h_{1}$ and $e_{2}=r_{2}-h_{2}$. The positive controller gains $\kappa_{1}, \kappa_{2}, \kappa_{3}$, and $\kappa_{4}$ are chosen such $\kappa_{1}>2 \sqrt{\kappa_{2}}$ and $\kappa_{3}>2 \sqrt{\kappa_{4}}$.

The following proposition gives the main result of this section.

Proposition 2. The input-output feedback linearization controller controller (12) when applied to the quadruple tank process (1) guarantees the exponential convergence of the water levels $h_{1}$ and $h_{2}$ to their desired values $h_{1}^{o}$ and $h_{2}^{o}$ respectively as $t$ tends to infinity. Moreover, the controller (12) guarantees the boundedness of the water levels $h_{3}(t)$ and $h_{4}(t)$ (i.e., $0 \leq h_{3}(t) \leq q_{1}$, and $0 \leq h_{4}(t) \leq q_{2}$ for some positive constants $q_{1}$ and $\left.q_{2}\right)$.

Proof: The application of the controllers $v_{1}$ and $v_{2}$ given by (12) to the dynamical model of the quadruple tank process (1) results in the following error dynamics:

$$
\dot{e}_{1}=-\kappa_{1} e_{1}-\kappa_{2} \int_{0}^{t} e_{1} d \dot{t}, \quad \dot{e}_{2}=-\kappa_{3} e_{2}-\kappa_{4} \int_{0}^{t} e_{2} d \dot{t}
$$

The error dynamics (13) can be written as $\ddot{e}_{1}+\kappa_{1} \dot{e}_{1}+\kappa_{2} e_{1}=0$ and $\ddot{e}_{2}+\kappa_{3} \dot{e}_{2}+\kappa_{4} e_{2}=0$. By choosing $\kappa_{1}>2 \sqrt{\kappa_{2}}$ and $\kappa_{3}>2 \sqrt{\kappa_{4}}$, we are guaranteed that the characteristic equations $s^{2}+\kappa_{1} s+\kappa_{2}=0$ and $s^{2}+\kappa_{3} s+\kappa_{4}=0$ have negative real roots. In this case, the solutions of (13) are given by:

$$
e_{1}(t)=c_{1} \exp \left(-\lambda_{1} t\right)+c_{2} \exp \left(-\lambda_{2} t\right), \quad e_{2}(t)=c_{3} \exp \left(-\lambda_{3} t\right)+c_{4} \exp \left(-\lambda_{4} t\right)
$$

where $-\lambda_{i}(i=1, \ldots, 4)$ are the roots of the above characteristic equations, and $c_{i}(i=1, \ldots, 4)$ are constants which depend on the initial conditions and the values of the $\lambda_{i}$. Therefore, the errors $e_{1}$ and $e_{2}$ exponentially converge to zero as t tends to infinity (i.e., the water levels $h_{1}$ and $h_{2}$ exponentially converge to their desired levels $h_{1}^{o}$ and $h_{2}^{o}$ respectively as t tends to infinity). Using equations (12)-(14), it can be shown that $\dot{h}_{3}$ and $\dot{h}_{4}$ in (1) can be written as follows:

$$
\dot{h}_{3}=-p_{7} \sqrt{h_{3}}-\left(p_{5} p_{8} / p_{6}\right) \sqrt{h_{4}}+m_{1}(t), \quad \dot{h}_{4}=-\left(p_{2} p_{10} / p_{3}\right) \sqrt{h_{3}}-p_{9} \sqrt{h_{4}}+m_{2}(t)
$$


where,

$$
\begin{gathered}
m_{1}(t)=\left(p_{8} / p_{6}\right)\left(c_{3} \lambda_{3} \exp \left(-\lambda_{3} t\right)+c_{4} \lambda_{4} \exp \left(-\lambda_{4} t\right)+p_{4} \sqrt{h_{2}^{o}-c_{3} \exp \left(-\lambda_{3} t\right)-c_{4} \exp \left(-\lambda_{4} t\right)}\right) \\
m_{2}(t)=\left(p_{10} / p_{3}\right)\left(c_{1} \lambda_{1} \exp \left(-\lambda_{1} t\right)+c_{2} \lambda_{2} \exp \left(-\lambda_{2} t\right)+p_{1} \sqrt{h_{1}^{o}-c_{1} \exp \left(-\lambda_{1} t\right)-c_{2} \exp \left(-\lambda_{2} t\right)}\right)
\end{gathered}
$$

It can be shown that $m_{1}(t)$ and $m_{2}(t)$ are bounded from above for all $t \geq 0$, i.e., $m_{1}(t) \leq \bar{m}_{1}$ and $m_{2}(t) \leq \bar{m}_{2}$ where $\bar{m}_{1}$ and $\bar{m}_{2}$ are some constants. Hence, it can be concluded that $\bar{h}_{3} \leq$ $-p_{7} \sqrt{h_{3}}+\bar{m}_{1}$ and $\dot{h}_{4} \leq-p_{9} \sqrt{h_{4}}+\bar{m}_{2}$.

Consider the differential equation $\dot{\hat{h}}_{3}=-p_{7} \sqrt{\hat{h}_{3}}+\bar{m}_{1}$ with the initial value $\hat{h}_{3}(0)=h_{3}(0)$. Let Lyapunov function candidate $V_{1}\left(\hat{h}_{3}\right)=\hat{h}_{3}^{2}$, then $\dot{V}_{1}=-2 \hat{h}_{3}\left(p_{7} \sqrt{\hat{h}_{3}}-\bar{m}_{1}\right)$. Notice that $\dot{V}_{1}$ is negative when $\sqrt{\hat{h}_{3}}>\left(\bar{m}_{1} / p_{7}\right)$ (which corresponds to $\left.V_{1}>\left(\bar{m}_{1} / p_{7}\right)^{4}\right)$. This means that all solutions starting such that $V_{1}(0)>\left(\bar{m}_{1} / p_{7}\right)^{4}$ will decrease monotonically but will never go below the line $V_{1}=\left(\bar{m}_{1} / p_{7}\right)^{4}$, while all solutions starting such that $V_{1}(0) \leq\left(\bar{m}_{1} / p_{7}\right)^{4}$ will increase monotonically but they will never cross the line $V_{1}=\left(\bar{m}_{1} / p_{7}\right)^{4}$ because $\dot{V}_{1}$ is negative for $V_{1}>\left(\bar{m}_{1} / p_{7}\right)^{4}$. Therefore, we can conclude that $V_{1} \leq \max \left\{V_{1}(0),\left(\bar{m}_{1} / p_{7}\right)^{4}\right\}$ or $\hat{h}_{3} \leq$ $\max \left\{\hat{h}_{3}(0),\left(\bar{m}_{1} / p_{7}\right)^{2}\right\}$. The comparison principle [22] leads us to conclude that $h_{3}(t) \leq \hat{h}_{3}(t)$. Since $h_{3}(t) \geq 0$, we can conclude that $0 \leq h_{3}(t) \leq q_{1}:=\max \left\{h_{3}(0),\left(\bar{m}_{1} / p_{7}\right)^{2}\right\}$. Similar arguments can be used to conclude that $0 \leq h_{4}(t) \leq q_{2}:=\max \left\{h_{4}(0),\left(\bar{m}_{2} / p_{9}\right)^{2}\right\}$.

Therefore, it can be concluded that the proposed controller (12) guarantees the exponential convergence of $h_{1}$ and $h_{2}$ to their desired values as well as the boundedness of $h_{3}$ and $h_{4}$.
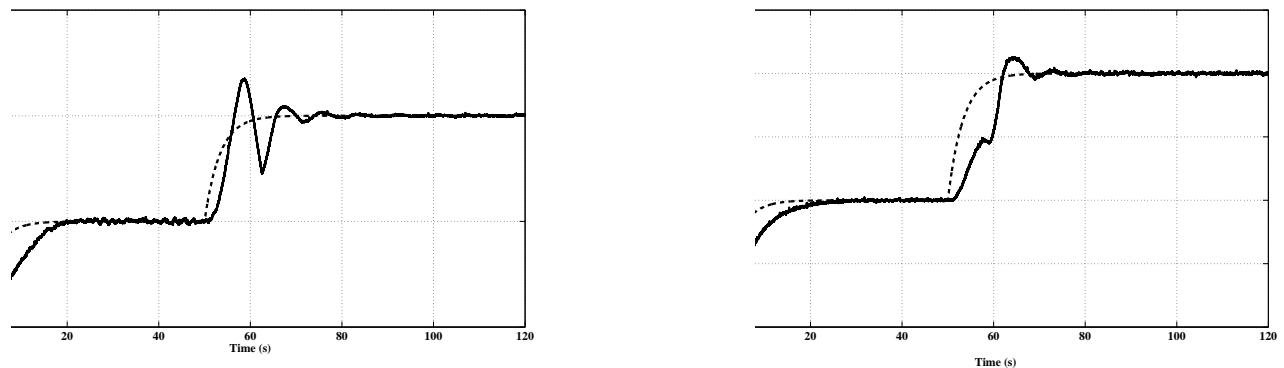

Figure 3: The water levels of tank 1 (solid: left) and tank 2 (solid: right) when using the gain scheduling controller. The references $r_{1}$ and $r_{2}$ are depicted using the dashed lines
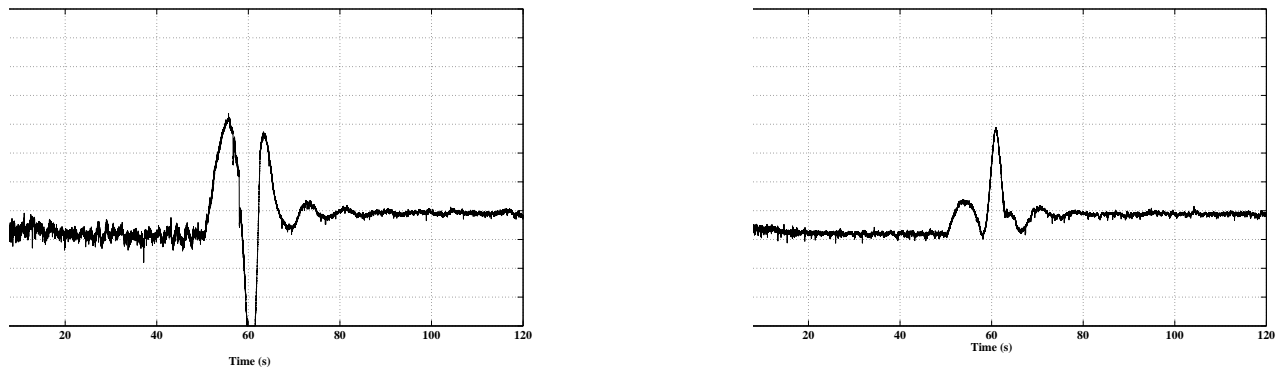

Figure 4: The inputs $v_{1}$ (left) and $v_{2}$ (right) when using the gain scheduling controller 

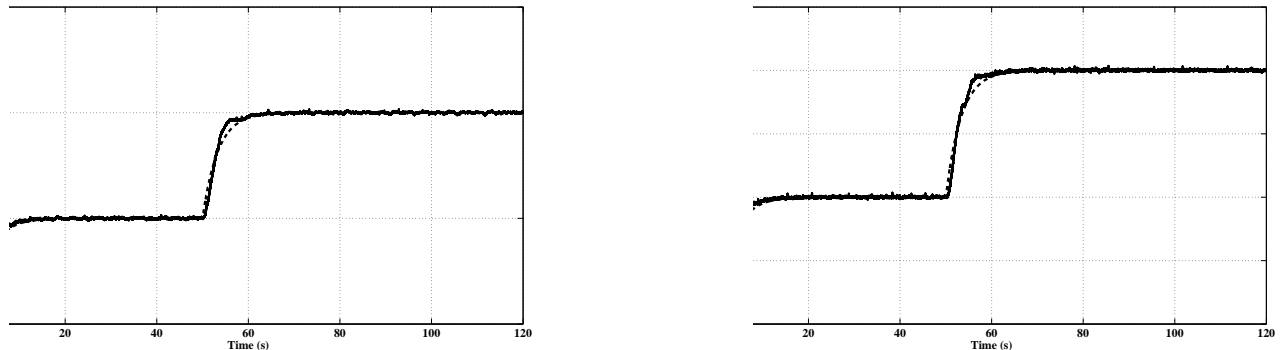

Figure 5: The water levels of tank 1 (solid: left) and tank 2 (solid: right) when using the linear parameter varying controller. The references $r_{1}$ and $r_{2}$ are depicted using the dashed lines
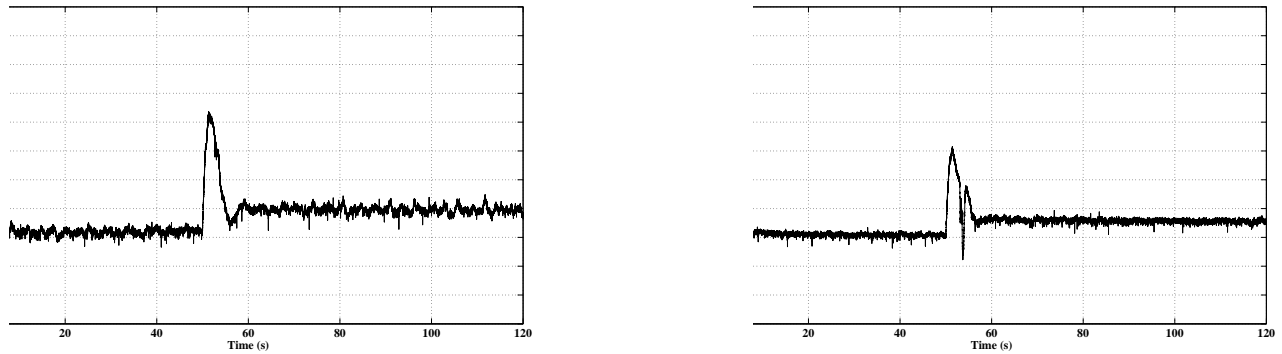

Figure 6: The inputs $v_{1}$ (left) and $v_{2}$ (right) when using the linear parameter varying controller

\section{$7 \quad$ Experimental results}

The three proposed control schemes are implemented using an experimental setup of the quadruple tank process manufactured by Quanser Consulting Inc. [24]. The physical parameters of the quadruple tank system are as follows: $A_{1}=A_{2}=A_{3}=A_{4}=15.5179 \mathrm{~cm}^{2}, a_{1}=a_{2}=$ $a_{3}=a_{4}=0.1781 \mathrm{~cm}^{2}, g=981 \mathrm{~cm} / \mathrm{s}^{2}, k_{1}=k_{2}=3.3 \mathrm{~cm}^{3} / V s, \gamma_{1}=0.66$, and $\gamma_{2}=0.75$. The sampling rate of the process is $10^{-3}$ seconds. The reference signal $r=\left[r_{1}, r_{2}\right]^{T}$ is chosen such that $r_{1}$ changes its amplitude from $5 \mathrm{~cm}$ to $10 \mathrm{~cm}$ at $t=50 \mathrm{sec}$ and $r_{2}$ changes its amplitude from $4 \mathrm{~cm}$ to $8 \mathrm{~cm}$ at $t=50 \mathrm{sec}$.

At first, the gain scheduling controller given by (7) is used to regulate the output $y$ to the operating point $\left(h_{1}^{o^{i}}, h_{2}^{o^{i}}\right)=(5 \mathrm{~cm}, 4 \mathrm{~cm})$ and then to the operating point $\left(h_{1}^{o+1}, h_{2}^{o^{i+1}}\right)=$ $(10 \mathrm{~cm}, 8 \mathrm{~cm})$. The controller gains, at each operating point, are designed such that the closed loop poles lie inside the region shown in Fig. 2 where $\tau=0.03, \zeta=20^{\circ}$ and $\rho=2$. The experimental results are shown in Figs. 3-4. Fig. 3 shows the water levels in tanks 1 and 2, while Fig. 4 shows the input voltages to pump 1 and pump 2. It can be seen from the figures that the water levels $h_{1}$ and $h_{2}$ track the desired reference signal $r$. However, the water level $h_{1}$ exhibits a percent overshoot of about $20 \%$ and a settling time of about 20 seconds while the water level $h_{2}$ exhibits a percent overshoot of about $6 \%$ and a settling time of about 20 seconds. Also, the input voltages stay within reasonable ranges. It should be noted that the performance of the system can be further improved through proper tuning of the parameters of the controller.

Secondly, the linear parameter varying controller given by (9) is designed and implemented such that the system is stable over the whole operating range $0 \leq h_{i} \leq 30 \mathrm{~cm}$; the closed loop poles are located inside the region shown in Fig. 2 where $\tau=0.03, \zeta=20^{\circ}$ and $\rho=2$. The experimental results are shown in Figs. 5-6. Fig. 5 shows the water levels in tanks 1 and 2, while Fig. 6 shows the input voltages to pump 1 and pump 2. It can be seen from the figures that the 

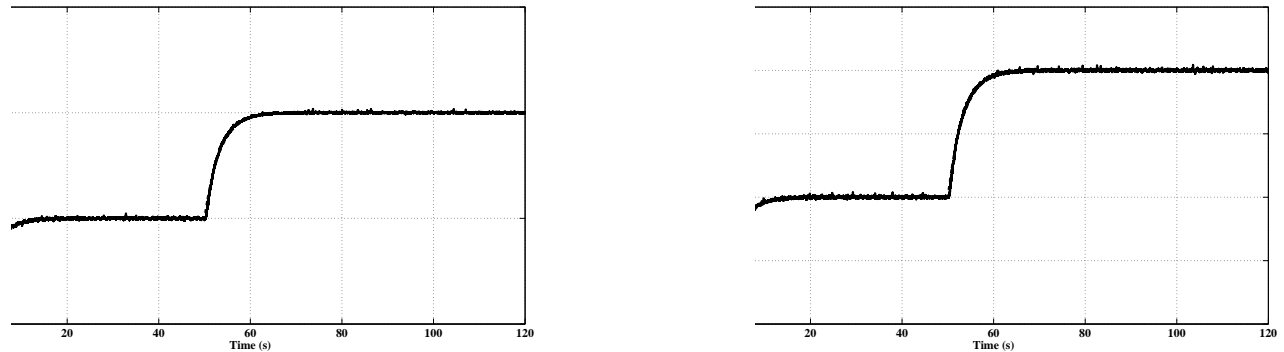

Figure 7: The water levels of tank 1 (solid: left) and tank 2 (solid: right) when using the inputoutput feedback linearization controller. The references $r_{1}$ and $r_{2}$ are depicted using the dashed lines
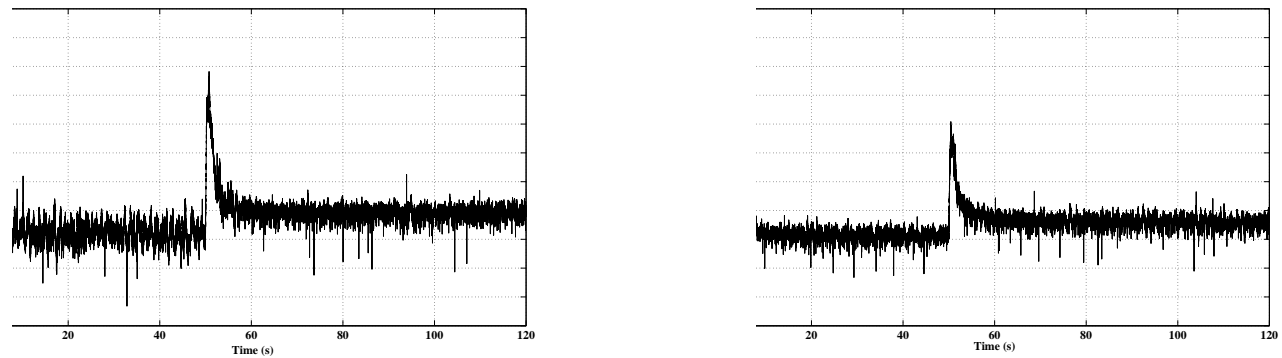

Figure 8: The inputs $v_{1}$ (left) and $v_{2}$ (right) when using the input-output feedback linearization controller

water levels $h_{1}$ and $h_{2}$ track the desired reference signal $r$ with no overshoot and a settling time of about 10 seconds for both $h_{1}$ and $h_{2}$. Also, it can be seen that the input voltages stay within reasonable ranges.

Thirdly, the input-output feedback linearization controller given by (12) is applied to the quadruple tank process. The parameters of the controller are taken to be $\kappa_{1}=\kappa_{3}=3$ and $\kappa_{2}=\kappa_{4}=1$. The experimental results are shown in Figs. 7-8. Fig. 7 shows the water levels in tanks 1 and 2, while Fig. 8 shows the input voltages to pump 1 and pump 2. It can be seen from the figures that the water levels $h_{1}$ and $h_{2}$ track the desired reference signal $r$ very well. Also, the input voltages stay within reasonable ranges (but display a bit more chattering).

To compare the performances of the proposed control schemes, the errors $e_{1}=r_{1}-h_{1}$ and $e_{2}=r_{2}-h_{2}$ are plotted in Fig. 9. It is clear from this figure that all the errors converge to zero. However, the errors for the input-output feedback linearization controller are less than the errors of the other two controllers. In addition, the figures show that the linear parameter varying controller gave better results than the gain scheduling controller. Furthermore, it is noted that the input-output feedback linearization controller can be implemented easily. 

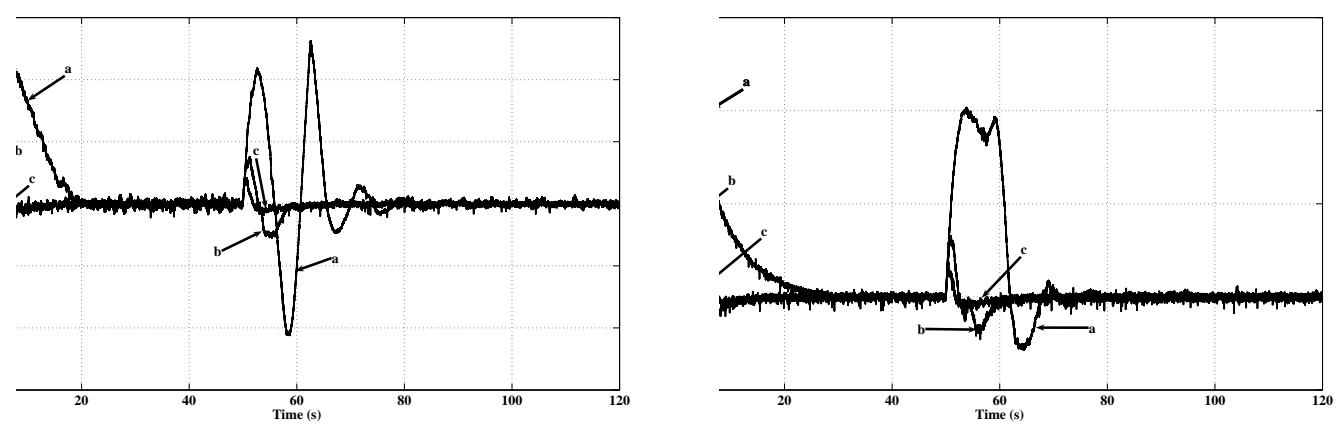

Figure 9: The errors $e_{1}$ (left) and $e_{2}$ (right) when using the gain scheduling controller (a), the linear parameter varying controller (b) and the input-output feedback linearization controller (c) 


\section{Conclusion}

A gain scheduling controller, a linear parameter varying controller, and an input-output feedback linearization controller are proposed for the quadruple tank process. At first, we propose to use a gain scheduling controller. However, this controller does not ensure the stability and the performance of the closed loop system over the whole operating range. Therefore, a linear parameter varying controller which guarantees the stability and the performance of the system over the desired operating range is proposed to control the process. However, the design of the linear parameter varying controller is quite complicated. Therefore, to reduce the design/implementaion complexity, an input-output feedback linearization controller is derived for the process. Experimental results are presented for the three control schemes. The implementation results indicate that the three proposed control schemes work well and are able to regulate the output of the system to its desired value. However, the implementation results indicate that the inputoutput feedback linearization controller gave the better performance in comparison with the other two controllers. Future research will address the design of fault-tolerant control schemes for the quadruple tank process.

\section{Bibliography}

[1] K.H. Johansson, "The quadruple-tank process: a multivariable laboratory process with an adjustable zero," IEEE Trans. Control Syst. Technol., vol. 8, pp. 456-465, 2008.

[2] B. Moaveni, A. Khaki-Sedigh, "Input-output pairing for nonlinear multivariable systems," $J$. Appl. Sci., vol. 22, pp. 3492-3498, 2007.

[3] F. Garelli, R.J. Mantz, H. De Battista, "Limiting interactions in decentralized control of MIMO systems," J. Process Control, vol. 16, pp. 473-483, 2006.

[4] K.J. Astrom, K.H. Johansson, Q.G. Wang, "Design of decoupled PI controllers for two-by-two systems," IEE Proc. Control Theory Appl., vol. 149, pp. 74-81, 2002.

[5] E.P. Gatzke, E.S. Meadows, C. Wang, F.J. Doyle III, "Model based control of a four-tank system," Comput. Chem. Eng., vol. 24, pp. 1503-1509, 2000.

[6] M. Mercangoz, F.J. Doyle III, "Distributed model predictive control of an experimental fourtank system," J. Process Control, vol. 17, pp. 297-308, 2007.

[7] D. Henriksson, A. Cervin, J. Akesson, K. Arzen, "On dynamic real-time scheduling of model predictive controllers," 41st IEEE Conference on Decision and Control, Las Vegas, pp. 1325$1330,2002$.

[8] S.M. Mahdi Alavi, A. Khaki-Sedigh, B. Labibi, M.J. Hayes, "Improved multivariable quantitative feedback design for tracking error specifications," IET Control Theory Appl., vol. 1, pp. 1046-1053, 2007.

[9] R. Vadigepalli, E.P. Gatzke, F. Doyle III, "Robust control of a multivariable experimental four-tank system," Ind. Eng. Chem. Res., vol. 40, pp. 1916-1927, 2001.

[10] R. Findeisen, F. Allgower, L.T. Biegler, Assessment and Future Directions of Nonlinear Model Predictive Control, Springer, Berlin, 2007. 
[11] N. Barhoumi, S. HadjSaid, F. M'sahli, "Constrained nonlinear model predictive control of hybrid dynamic systems," J. Auto. Syst. Eng., vol. 3, pp. 46-56, 2009.

[12] P.P. Biswasa, R. Srivastavaa, S. Raya, A.N. Samanta, "Sliding mode control of quadruple tank process," Mechatronics, vol. 19, pp. 548-561, 2009.

[13] B. Labibi, H.J. Marquez, T. Chen, "Decentralized robust output feedback control for control affine nonlinear interconnected systems," J. Process Control, vol. 19, pp. 865-878, 2009.

[14] F.D. Bianchi, R.J. Mantz, C.F. Christiansen, "Multivariable PID control with set-point weighting via BMI optimisation," Automatica, vol. 44, pp. 472-478, 2008.

[15] W.J. Rugh, J.S. Shamma, "Research on gain scheduling," Automatica, vol. 36, pp. 14011425, 2000.

[16] R.A. Hyde, K. Glover, "The application of scheduled $H_{\infty}$ controllers to a VSTOL aircraft," IEEE Trans. Autom. Control, vol. 38, pp. 1021-1039, 1993.

[17] N. Aouf, D.G. Bates, I. Postlethwaite, B. Boulet, "Scheduling schemes for an integrated flight and propulsion control system," Control Eng. Pract., vol. 10, pp. 685-696, 2002.

[18] J.S. Shamma, M. Athans, "Guaranteed properties of gain scheduled control for linear parameter varying plants," Automatica, vol. 3, pp. 559-564, 1991.

[19] P. Apkarian, P. Gahinet, "A convex characterization of gain-scheduled $\mathrm{H}_{\infty}$ controllers," IEEE Trans. Autom. Control, vol. 5, pp. 853-864, 1995.

[20] M. Chilali, P. Gahinet, "H$H_{\infty}$ design with pole placement constraints: an LMI approach," IEEE Trans. Autom. Control, vol. 41, pp. 358-367, 1996.

[21] P. Apkarian, H.D. Tuan, "Parameterized LMIs in control theory," SIAMJ. Control Optim., vol. 4, pp. 1241-1264, 2000.

[22] H.K. Khalil, Nonlinear Systems, Prentice-Hall, New Jersey, 2002.

[23] J.J.E. Slotine, W. Li, Applied Nonlinear Control, Prentice-Hall, New Jersey, 1991.

[24] J. Apkarian, Coupled Water Tank Experiments Manual, Quanser Consulting Inc., Canada, 1999.

[25] P. Gahinet, A. Nemirovski, A. Laub, M. Chilali, LMI Control Toolbox User's Guide, The MathWorks Inc., Natick, MA, 1995.

[26] G.E. Forsythe, M.A. Malcolm, C.B. Moler, Computer Methods for Mathematical Computations, Prentice-Hall, New Jersey, 1977. 